\title{
Probabilistic Forecast of Electricity Price based on Adaboost_RBF method
}

\author{
Junli $\mathrm{Wu}^{1, \mathrm{a}}$, Zhen $\mathrm{Wu}^{1, \mathrm{~b}}$, Jinhua Huang ${ }_{\mathrm{e}}^{1, \mathrm{c}}$, HouYin Long ${ }^{1, \mathrm{~d}}$, and Chengjin $\mathrm{Ye}^{1,}$ \\ ${ }^{1}$ State Grid Zhejiang Economic Research institute, Hangzhou 310000, China. \\ aWujunli_hust@163.com, b wuzhen_zj@sina.com, ${ }^{\mathrm{c}}$ Huangjinhua@sina.com, \\ d Longhouyin @sina.com ${ }^{\mathrm{e}}$ Yechengjin@sina.com
}

Keywords: electricity price, probabilistic forecast, Adaboost_RBF, prediction interval.

\begin{abstract}
Accurate and reliable electricity price forecasting is essential for market participants to make various decisions in the deregulated electricity market. However, due to the time-variant and nonstationary of price, which is related to change of market competitors' strategies, predicting price accurately in advance is rather difficult. Therefore, probabilistic interval forecast instead of traditional point forecast can be of great significance to make bidding strategies. In this paper, a hybrid approach for probabilistic forecast is proposed with two-stage formulation: 1) An improved RBF NNs based on Adaboost algorithm (Adaboost_RBF) is proposed for point forecast of price. 2) Prediction interval can be obtained according to the statistical distribution of price forecast error. Effectiveness and reliability of proposed model is validated through case studies from Australian electricity market by comparing with existing methods such as RBF neural network and ARMA.
\end{abstract}

\section{Introduction}

The electric power industries of many countries all over the world have experienced enormous changes and deregulated electricity market is built in the past few decades[1]. In electricity market, price is significant to guide the market behaviors of participants, such as demand side participation of consumers and bidding strategies of suppliers. Electricity consumers will actively react to electricity price through intelligent management of electric power consumption for economy and environmental benefits[2]. Moreover, market suppliers can facilitate bidding schemes and short-term operation according to forecasted price. Therefore, accurate electricity price forecast becomes more and more essential to the operation of electricity market. However, It is quite difficult to forecast future price value accurately for the reason that price is a nonstationary stochastic time series with high uncertainty[3]. Accordingly, probabilistic forecast becomes more meaningful by offering prediction intervals to quantify the uncertainties of forecast price. The prediction intervals can be applied to estimate the risks of the bidding strategies of market suppliers.

Accurate electricity price probabilistic forecast has two major challenges. 1) It is difficult to forecast exact value of future price, because the electricity price is nonstationary and highly volatile. 2) Besides the forecast value, the probability distribution of price forecast error should be established to analyze the uncertainty of forecast price and estimate prediction interval. However, the probability distribution of forecast variance is more challenging because the variances are extremely different associated with various forecast values.

Most researchers in this field pay more attention to point forecast of electricity price, by far. The traditional techniques such as regression model [4], autoregressive integrated moving average (ARIMA)[5,6] is proposed to solve the point forecast of electricity price. Wavelet technique is employed to improve the forecast performance [7]. However, these methods cannot represent nonlinear characteristics of price. AI-based methods such as neural networks (NNs) have been developed by many researchers for price forecast to overcome the restrictions of linear time series model [8-13]. Moreover, Support machine vector (SVM) is applied to achieve favorable results. Nevertheless, these methods cannot forecast prediction interval of price. 
Electricity price forecast errors are unavoidable and significant to analyze bidding risks in market. Therefore, instead of traditional point forecast techniques, probabilistic forecast attracts more attention than as it can estimate the uncertainty of forecasted price by offering prediction intervals. Market participants can make reasonable decisions and prepare for the worst case. ARIMA model is proposed to forecast price value and associated forecast errors with relatively good performance [3]. In order to overcome the limited ability of accurately forecast nonlinear electricity price, literature [14] proposes generalized autoregressive conditional heteroscedasticity model (GARCH) as an effective method to model heteroscedasticity of electricity price series and forecast time-changing variance. In addition, Literature[15] proposes nonlinear conditional heteroscedastic forecast (NCHF) model based on SVM for interval prediction. Integrated adaptive learning method combined with neural networks and extended Kalman filter (EKF) for price prediction and corresponding confidence interval (CI) [16]. However, most related work do not analyze statistical characteristic of electricity price forecast error. Without statistical analysis, prediction interval cannot be accurately obtained.

The aim of paper is to provide a novel method for prediction interval forecast of day-ahead electricity price with excellent performance. A hybrid method is proposed for probabilistic prediction of electricity price based on improved neural networks and statistical analysis. NNs are widely applied in electricity forecast problem as its excellent approximation ability [8-11]. Nevertheless, because of the drawbacks of classical NNs such as local minima, time consuming training process and overfitting, traditional NNs cannot achieve satisfactory prediction result. The performance of NNs is increased obviously based on radial basis function (RBF)[12-13]. Adaboost is a novel learning algorithm, which can improve learning performance with simple and easy implementation [17]. Therefore, the paper proposes improved RBF neural networks based on Adaboost algorithm (Adaboost_RBF) for point prediction of electricity price. Subsequently, through statistically analyze the price forecast error, the paper use Normal distribution to describe probability distribution of the forecast error associated with Adaboost_RBF prediction method in electricity market. The proposed prediction interval method is tested on Australian electricity market by comparing reliability and sharpness indexes with existing methods. The result demonstrates the effectiveness and reliability of proposed method, which can provide market participants available tool to forecast market price prediction interval with high operability.

The organization of paper is as follows: In Section II, the formulation and construction of Adaboost_RBF are firstly proposed. Section III introduces the framework of prediction interval construction. In Section IV, Numerical studies of Australian National Electricity Market are conducted to demonstrate effectiveness and reliability of proposed model. Finally, the paper draws the conclusions in Section VI.

\section{Adaboost_RBF for electricity price prediction}

\subsection{RBF Neural networks}

Neural networks (NNs) have been widely used to forecast electricity price as its approximation ability. However, the traditional NNs have drawbacks such as local minima and overfitting, which can be overcome through radial basis function (RBF). RBF NNs consists of input layer, hidden layer and output layer. The output of RBF NNs is given by

$$
F\left(\mathbf{x}_{i}\right)=\sum_{i=1}^{M} w_{j} G\left(\left\|\mathbf{x}_{i}-\mathbf{c}_{j}\right\|\right)
$$

Where $\mathbf{x}_{i}$ is the input vector, $\mathbf{c}_{j}$ is the center location of hidden layer node $\mathrm{j},\left\|\mathbf{x}_{i}-\mathbf{c}_{j}\right\|$ is the Euclidean distance between $\mathbf{x}_{i}$ and $\mathbf{c}_{j}, G(\cdot)$ is the radial basis function, $M$ is the number of hidden layer nodes, $w_{j}$ is the transfer weight from hidden layer to output layer. The hidden layer nodes generate local response to the input information through basis function including gauss function, reflected sigmoidal function and Inverse multiquadrics function. The output of hidden layer node is associated with distance between center of hidden layer node and input vector. 
In this paper, extreme learning machine (ELM)[18] is employed as training algorithm because of several advantages, such as fast convergent rate and overcoming local minima. In ELM, the center location $\mathbf{c}_{j}$ and hidden layer weights $w_{j}$ are randomly assigned and adjusted during training process.

\subsection{Adaboost_RBF}

Adaboost is an effective algorithm to solve the problem of regression and classification among machine learning field [17]. Adaboost can establish a strong learning machine by combining multiple weak learning machines, which are trained by the same training set. As Adaboost can improve the learning performance of weak learning machine, an improved RBF NNs based on Adaboost algorithm (Adaboost_RBF) is proposed for point forecast of electricity price in this paper.

The training process of Adaboost is introduced as follows:

1) Firstly, obtain the training set $\mathbf{S}=\left\{\left(\mathbf{x}_{i}, P_{i}\right)\right\}_{i=1}^{N}$. it can be obviously found that there is strong correlation between electricity price and load demand through the research of literature [19]. The input data $\mathbf{x}_{i}$ include historical price data and load demands, while the output data $P_{i}$ is the prediction target.

2) Initialize the training sample $\left(\mathbf{x}_{i}, P_{i}\right)$ weight $w_{i}=1 / N$ and iteration number $t=1$.

3) Select the training data $\mathbf{S}_{t}=\left\{\left(\mathbf{x}_{i}, P_{i}\right)\right\}_{i=1}^{N_{t}}$ from training set $\mathbf{S}$ according to the weight of training sample. The higher weight $w_{i}$ indicates that training sample $\left(\mathbf{x}_{i}, P_{i}\right)$ is more likely to be selected in training data set $\mathbf{S}_{t}$. $N_{t}$ is the number of training sample in $\mathbf{S}_{t}$.

4) Train the RBF neural network $N N_{t}$ by using ELM.

5) Calculate the training error by equation (2-3),

$$
\begin{aligned}
& \varepsilon_{t, i}=\left|N N_{t}\left(\mathbf{x}_{i}\right)-P_{i}\right| \\
& \varepsilon_{t}=\sum_{i=1}^{N_{t}} w_{i} \cdot \varepsilon_{t, i}
\end{aligned}
$$

Where $N N_{t}\left(\mathbf{x}_{i}\right)$ is the output of RBF neural network. $\varepsilon_{t, i}$ is the training error of sample $\left(\mathbf{x}_{i}, P_{i}\right) . \varepsilon_{t}$ is the error of $N N_{t}$.

6) Set the weight of RBF neural network

$$
\alpha_{t}=\frac{1}{2} \ln \left(\frac{1-\varepsilon_{t}}{\varepsilon_{t}}\right)
$$

7) Adjust the weight of training sample according to the training error.

$$
\begin{aligned}
& \beta_{t}=\frac{\varepsilon_{t}}{1-\varepsilon_{t}} \\
& w_{t, i}=w_{t-1, i} \beta_{t}^{1-\varepsilon_{t, i}} / Z_{t} \\
& Z_{t}=\sum_{i=1}^{N} w_{t-1, i} \beta_{t}^{1-\varepsilon_{t, i}}
\end{aligned}
$$

Where $Z_{t}$ is the normalization coefficient to ensure $\sum_{i=1}^{N} w_{t, i}=1$

8) Repeat steps 3-7 and set $t=t+1$ until $t=t_{\max }$, where $t_{\max }$ is the maximum number of iteration. Then obtain strong learning machine $H\left(\mathbf{x}_{i}\right)$

$$
H\left(\mathbf{x}_{i}\right)=\sum_{i=1}^{N_{t}} \alpha_{t} N N_{t}\left(\mathbf{x}_{i}\right)
$$




\section{Framework of prediction interval construction}

The uncertainties of RBF neural network forecast model based on Adaboost is mainly caused by noise of training data due to the stochastic characteristics. The electricity price forecast target can be expressed as

$$
P_{i}=H\left(\mathbf{x}_{i}\right)+\varepsilon\left(\mathbf{x}_{i}\right)
$$

Generally, forecast errors always of electricity price follow the normal distribution. With the 100(1- $\alpha) \%$ confidence level, prediction interval $\left[L^{\alpha}\left(\mathbf{x}_{i}\right), U^{\alpha}\left(\mathbf{x}_{i}\right)\right]$ can be obtained by equations $(10-11)$.

$$
\begin{aligned}
& L^{\alpha}\left(\mathbf{x}_{i}\right)=N N_{t}\left(\mathbf{x}_{i}\right)-Z_{1-\alpha / 2} \\
& U^{\alpha}\left(\mathbf{x}_{i}\right)=N N_{t}\left(\mathbf{x}_{i}\right)+Z_{1-\alpha / 2}
\end{aligned}
$$

Where $z_{1-\alpha / 2}$ is the critical value of the normal distribution depending on the expected confidence level $100(1-\alpha) \%$. The probability of prediction target $P_{i}$ expected to lie in prediction interval.

Generally, the construction process of prediction interval consists of two steps. Fistrly, the point forecast of electricity price can be calculated by Adaboost_RBF. Secondly, with the quantified uncertainty model of forecast error, the prediction intervals can be obtained by equation (10) and (11).

\section{Literature References}

\subsection{Introduction of Case}

The paper applies the data of Australian National Electricity Market (ANEM) to validate the efficiency of the proposed forecast model. In ANEM, market clearing price is obtained by averaging 30 minutes dispatch prices based on bidding schedule of generators and consumers. The electricity price data cover the period from July 2013 to December 2014. In order to evaluate the efficiency of the proposed method, the results of proposed approach are compared with the RBF NNs and ARMA.

\subsection{Performance Evaluation}

In this paper, reliability and sharpness are applied as comprehensive indices for prediction interval evaluation[2].Reliability: The reliability is the key evaluation index about the correctness of the approximated prediction interval. According to the definition of prediction interval, the probability of electricity price in prediction interval should be expected to nominal probability $100(1-\alpha) \%$, which is termed as prediction interval nominal confidence(PINC)[2]. The actual coverage probability is represented as prediction interval coverage probability (PICP), which can reflect degree of reliability of prediction interval. PICP is defined as

$$
P I C P=\frac{1}{N} \sum_{i=1}^{N} M_{i}^{(\alpha)}
$$

Where $M_{i}^{(\alpha)}$ is the marker of PICP, given by

$$
M_{i}^{\alpha}= \begin{cases}1 & P_{i} \in\left[L^{\alpha}\left(\mathbf{x}_{i}\right), U^{\alpha}\left(\mathbf{x}_{i}\right)\right] \\ 0 & P_{i} \in\left[L^{\alpha}\left(\mathbf{x}_{i}\right), U^{\alpha}\left(\mathbf{x}_{i}\right)\right]\end{cases}
$$

The obtained prediction interval should be close to the corresponding PINC. Consequently, average coverage error (ACE), the difference between PINC and PICP, is applied to assess reliability of prediction interval.

$$
A C E=P I C P-P I N C
$$

The absolute ACE value is expected to be close to zero for the high reliability of prediction interval.

Sharpness: There are high correlation between reliability and width of prediction interval. With increasing width of prediction interval, high reliability can be easily obtained. However, it is meaningless for market participants to evaluate the risk. Therefore, interval score is employed to overall performance of prediction interval for sharpness perspective [20]. Width of prediction interval is given by 


\subsection{Comparison Results}

$$
W^{\alpha}\left(\mathbf{x}_{i}\right)=U^{\alpha}\left(\mathbf{x}_{i}\right)-L^{\alpha}\left(\mathbf{x}_{i}\right)
$$

In case study, the confidence levels of prediction interval of electricity price are set as $90 \%, 95 \%$ and 99\%. Two typical trading intervals (2:00-4:00 and 12:00-14:00) are selected to test the forecast models, which involves the peak load and low load in daily operation. The evaluation index including PICP and ACE are applied to evaluate the performance of the proposed approach and other two benchmark methods, and the results are given in Table I and Table II.

As seen in Tables I-IV, the proposed method performs better than ARMA and RBF method. PICPs of the proposed hybrid method are remarkably close to the related nominal confidence level. Moreover, the absolute ACEs of the proposed method are smaller than the other two methods, which indicate the reliability is more satisfactory than the other two methods. Overall, the proposed method provides the best predictive effect comparing with the other two benchmarks.

Table I Evaluation Results OF Prediction Interval on Trading Interval (2:00-4:00)

\begin{tabular}{cccc}
\hline Confidence Level & Method & PICP & ACE \\
\hline \multirow{2}{*}{$90 \%$} & ARMA & $87.56 \%$ & $2.44 \%$ \\
& RBF & $88.21 \%$ & $1.79 \%$ \\
\multirow{2}{*}{$95 \%$} & Adaboost_RBF & $89.21 \%$ & $0.79 \%$ \\
& ARMA & $91.73 \%$ & $3.27 \%$ \\
\multirow{2}{*}{$99 \%$} & RBF & $96.59 \%$ & $-1.59 \%$ \\
& Adaboost_RBF & $94.57 \%$ & $0.43 \%$ \\
& ARMA & $96.31 \%$ & $2.69 \%$ \\
& RBF & $98.16 \%$ & $0.84 \%$ \\
& Adaboost_RBF & $99.31 \%$ & $-0.31 \%$
\end{tabular}

Table II Evaluation Results OF Prediction Interval on Trading Interval (12:00-14:00)

\begin{tabular}{cccc}
\hline Confidence Level & Method & PICP & ACE \\
\hline \multirow{3}{*}{$90 \%$} & ARMA & $86.59 \%$ & $3.41 \%$ \\
& RBF & $88.52 \%$ & $1.48 \%$ \\
& Adaboost_RBF & $89.65 \%$ & $0.35 \%$ \\
\multirow{3}{*}{$95 \%$} & ARMA & $92.16 \%$ & $2.84 \%$ \\
& RBF & $93.85 \%$ & $1.15 \%$ \\
& Adaboost_RBF & $95.33 \%$ & $-0.33 \%$ \\
$99 \%$ & ARMA & $96.11 \%$ & $2.89 \%$ \\
& RBF & $97.31 \%$ & $1.69 \%$ \\
& Adaboost_RBF & $86.59 \%$ & $3.41 \%$ \\
\hline
\end{tabular}

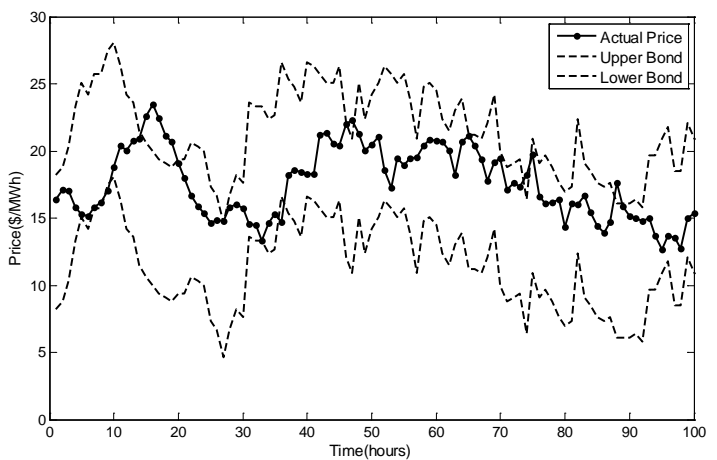

Fig. 1 Prediction interval of electricity price in trading Period 1 obtained by the proposed method (nominal confidence $=90 \%$ ) 


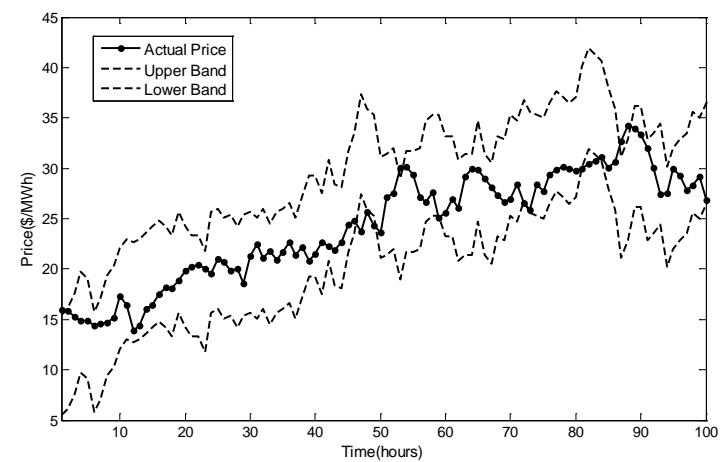

Fig.2 Prediction interval of electricity price obtained in trading Period 2 by the proposed method (nominal confidence $=90 \%$ )

To further demonstrate the effectiveness of the proposed method. The paper provides the prediction result of the proposed method in two trading period with fairly different variations in figs.1-2. The nominal confidence of prediction interval is set as $90 \%$. It can be obviously found that the actual electricity price is almost fit the prediction interval obtained by proposed method for two trading interval, which specifically demonstrate the favorable performance of the proposed method.

\section{Conclusion}

This paper presents a novel new method for probabilistic forecast of electricity price, which is combined with an Adaboost_RBF algorithm and prediction interval construction according to the statistical analysis. Reliable prediction interval can be estimated by the proposed method, which is significant to behaviors of participants in electricity market. Case studies of Australian National Electricity Market (ANEM) demonstrate the effectiveness and reliability of proposed method by comparing with two other methods.

\section{References}

[1]. González A M, Roque A M S, García-González J. Modeling and forecasting electricity prices with input/output hidden Markov models[J]. Power Systems, IEEE Transactions on, 2005, 20(1): 13-24.

[2]. Wan C, Xu Z, Wang Y, et al. A hybrid approach for probabilistic forecasting of electricity price[J]. Smart Grid, IEEE Transactions on, 2014, 5(1): 463-470.

[3]. Zhou M, Yan Z, Ni Y X, et al. Electricity price forecasting with confidence-interval estimation through an extended ARIMA approach[C]//Generation, Transmission and Distribution, IEE Proceedings-. IET, 2006, 153(2): 187-195.

[4]. Niimura T, Nakashima T. Deregulated electricity market data representation by fuzzy regression models[J]. Systems, Man, and Cybernetics, Part C: Applications and Reviews, IEEE Transactions on, 2001, 31(3): 320-326.

[5]. Contreras J, Espinola R, Nogales F J, et al. ARIMA models to predict next-day electricity prices[J]. Power Systems, IEEE Transactions on, 2003, 18(3): 1014-1020.

[6]. Nogales F J, Contreras J, Conejo A J, et al. Forecasting next-day electricity prices by time series models[J]. Power Systems, IEEE Transactions on, 2002, 17(2): 342-348.

[7]. Conejo A J, Plazas M A, Espinola R, et al. Day-ahead electricity price forecasting using the wavelet transform and ARIMA models[J]. Power Systems, IEEE Transactions on, 2005, 20(2): 1035-1042. 
[8]. Xu Z, Dong Z Y, Liu W Q. Neural network models for electricity market forecasting[J]. Neural networks applications in information technology and web engineering, 2005, 1: 233-245.

[9]. Catalão J P S, Mariano S, Mendes V M F, et al. Short-term electricity prices forecasting in a competitive market: a neural network approach[J]. Electric Power Systems Research, 2007, 77(10): 1297-1304.

[10]. Amjady N. Day-ahead price forecasting of electricity markets by a new fuzzy neural network[J]. Power Systems, IEEE Transactions on, 2006, 21(2): 887-896.

[11]. dos Santos Coelho L, Santos A A P. A RBF neural network model with GARCH errors: application to electricity price forecasting[J]. Electric Power Systems Research, 2011, 81(1): 74-83.

[12]. Amjady N, Daraeepour A, Keynia F. Day-ahead electricity price forecasting by modified relief algorithm and hybrid neural network[J]. Generation, Transmission \& Distribution, IET, 2010, 4(3): 432-444.

[13]. Meng K, Dong Z Y, Wong K P. Self-adaptive radial basis function neural network for short-term electricity price forecasting[J]. Generation, Transmission \& Distribution, IET, 2009, 3(4): 325-335.

[14]. Yun Z, Quan Z, Caixin S, et al. RBF neural network and ANFIS-based short-term load forecasting approach in real-time price environment[J]. Power Systems, IEEE Transactions on, 2008, 23(3): 853-858.

[15]. Garcia R C, Contreras J, Van Akkeren M, et al. A GARCH forecasting model to predict day-ahead electricity prices[J]. Power Systems, IEEE Transactions on, 2005, 20(2): 867-874.

[16]. Zhao J H, Dong Z Y, Xu Z, et al. A statistical approach for interval forecasting of the electricity price[J]. Power Systems, IEEE Transactions on, 2008, 23(2): 267-276.

[17]. Zhang L, Luh P B. Neural network-based market clearing price prediction and confidence interval estimation with an improved extended Kalman filter method[J]. Power Systems, IEEE Transactions on, 2005, 20(1): 59-66.

[18]. Lupaşcu C A, Tegolo D, Trucco E. FABC: retinal vessel segmentation using AdaBoost[J]. Information Technology in Biomedicine, IEEE Transactions on, 2010, 14(5): 1267-1274.

[19]. Huang G B, Zhu Q Y, Siew C K. Extreme learning machine: theory and applications[J]. Neurocomputing, 2006, 70(1): 489-501.

[20]. Motamedi A, Zareipour H, Rosehart W D. Electricity price and demand forecasting in smart grids[J]. Smart Grid, IEEE Transactions on, 2012, 3(2): 664-674. 